\title{
Initial experience with uniportal video-assisted thoracic surgery esophagectomy
}

\author{
Dania Nachira, Elisa Meacci, Maria Giovanna Mastromarino, Luca Pogliani, Edoardo Zanfrini, Amedeo \\ Iaffaldano, Leonardo Petracca-Ciavarella, Marco Chiappetta, Maria Teresa Congedo, Maria Letizia Vita, \\ Venanzio Porziella, Stefano Margaritora
}

Department of General Thoracic Surgery, Catholic University of Sacred Heart, Fondazione Policlinico Universitario “A.Gemelli”, Rome, Italy

Contributions: (I) Conception and design: D Nachira, E Meacci, MG Mastromarino; (II) Administrative support: S Margaritora; (III) Provision of study materials or patients: V Porziella, ML Vita, MT Congedo; (IV) Collection and assembly of data: M Chiappetta, L Petracca-Ciavarella, E Zanfrini, A Iaffaldano; (V) Data analysis and interpretation: D Nachira, E Meacci, MG Mastromarino; (VI) Manuscript writing: All authors; (VII) Final approval of manuscript: All authors.

Correspondence to: Dania Nachira, MD. Department of General Thoracic Surgery, Fondazione Policlinico Universitario "A.Gemelli”, Largo A. Gemelli, 8, 00168, Rome, Italy. Email: danynac@libero.it.

Background: Multiportal thoracoscopic approach is already a well standardized procedure for minimally invasive esophagectomy (MIE); conversely very few reports have been published about uniportal videoassisted thoracic surgery (VATS) technique till now. We present our preliminary experience with uniportal VATS esophagectomy, evaluating short-term outcomes as perioperative mortality, complications, oncological radicality, postoperative pain and cosmetic results.

Methods: From December 2016 to November 2017, the prospectively collected clinical data of 12 patients, who underwent uniportal VATS esophagectomy and reconstruction with a stomach conduit, according to McKeown technique, were reviewed and outcomes evaluated.

Results: The mean age of population was $60.67 \pm 8.61$ years. Ten $(83.3 \%)$ patients were males. The main histological type was a squamous cell carcinoma in six patients $(50 \%)$. No patient had a local recurrence. After $4.33 \pm 3.31$ months 10 patients $(83.3 \%)$ were alive with no evidence of disease; 2 (16.7\%) patients died of other causes. Two (16.7\%) patients developed an anastomotic leak (treated conservatively) and one (8.3\%) patient a chylothorax (which required a surgical treatment). The mean operative time of uniportal VATS esophagectomy was $104.67 \pm 20.66 \mathrm{~min}$. Mean number of thoracic nodes removed was $10.44 \pm 3.94$. Postoperative hospitalization was $15.73 \pm 14.29$ days (median of 9 days). The mean level of pain was $1.92 \pm 0.90$ in first postoperative day with a duration of $2.25 \pm 1.54$ days. Cosmetic result was $2.42 \pm 0.79$ on a 3-point scale.

Conclusions: Uniportal VATS esophagectomy seems to be a safe, feasible and effective alternative to multiportal VATS in terms of operative time, postoperative mortality, hospital stay and oncological outcomes. Less postoperative pain and better cosmetic results seem to be some advantages in favor of Uniportal VATS, however further studies with longer follow-up are claimed.

Keywords: Uniportal video assisted thoracoscopy (uniportal VATS); esophagectomy; esophageal cancer

Submitted Feb 06, 2018. Accepted for publication Mar 28, 2018.

doi: $10.21037 /$ jtd.2018.04.17

View this article at: http://dx.doi.org/10.21037/jtd.2018.04.17

\section{Introduction}

Esophageal cancer is an aggressive malignancy with a relatively poor prognosis. It represents the $6^{\text {th }}$ leading cause of cancer death and the $8^{\text {th }}$ most common cancer type worldwide. The 5-year survival is around $15-25 \%$, with best results in early stages due to timely diagnosis (1). Regarding gender distribution, esophageal cancer is about 2-4 times more frequent among males compared to 
females. Two main histological types exist, characterized by a different incidence and distribution in the world. Esophageal squamous cell carcinoma is typically localized in the upper-middle esophagus and it is the most frequent histological type worldwide, with an incidence of more than 100 cases/100,000 person-years in the so-called "Asian Esophageal Cancer Belt" (2). However, currently, in United States and Western Europe, there is a predominance of adenocarcinoma subtype, which is usually localized in the lower esophagus. Its incidence has been increasing by $39.6 \%$ for men and $37.5 \%$ for women in the last 5 years (1).

Superficial cancers and high-grade dysplasia of the esophagus may be treated by endoscopic therapy. Surgery remains the first choice in early disease (T1-T2N0M0), based on the principles of complete primary tumor resection and radical lymphadenectomy. A multimodality approach consisting of preoperative chemoradiation followed by surgery is indicated in operable patients with locally advanced esophageal cancer (T3-T4N0M0 and T1$\mathrm{T} 4 \mathrm{aN}+\mathrm{M} 0$ ) (3). Esophagectomy is a relatively high invasive surgery, involving two or three-field access, depending on preoperative clinical staging and location of the cancer, which may lead to several postoperative morbidities. Although mortality in oesophageal resection is less than $5 \%$ in high-volume centres, at least half of the patients undergone open esophagectomy (OE) are at risk for developing respiratory complications with consequent longer hospital stay (4). To improve surgical outcome and reduce surgical morbidity, minimally invasive esophagectomy (MIE) has been performed since 1990s', due to the evolution of surgical laparoscopic and thoracoscopic techniques and endoscopic instrumentation (5). Different hybrid and total minimally invasive surgical approaches have been developed; the most commonly performed technique consists of thoracoscopic oesophageal mobilization followed by laparoscopy or upper median laparotomy and cervical anastomosis (6).

It has been demonstrated that thoracoscopic esophagectomy is comparable to conventional standard surgery with open thoracotomy in terms of the operating time, amount of blood loss, and number of dissected lymph nodes $(7,8)$. Therefore, allowing similar longterm oncological outcomes as open surgery (9), MIE is advantageous in terms of decreased respiratory complications, short length of hospital stay and early relief from postoperative pain and rapid restoration of vital capacity, as long as it is carried out at institutions with accumulated clinical experience (3).

Surgical thoracic options for MIE include robotic- assisted esophagectomy (RE) and thoracoscopic-assisted esophagectomy (TE), and no consensus has been reached till now among experts about the best approach (10). The relatively small diffusion of robotic instrumentation associated with its longer operation time and its higher hospital costs make TE the mainstay of esophageal cancer treatment nowadays. However, among TE, controversies still exist concerning the patient's position (prone versus lateral decubitus) and the number of surgical port-sites [uniportal video-assisted thoracic surgery (VATS) versus multiportal VATS esophagectomy] $(10,11)$. Multiportal approach is a standardized procedure largely performed in several centers worldwide $(8,12,13)$; conversely only very few papers have been published about uniportal VATS technique (12-15).

In the current paper we report our preliminary experience with uniportal VATS esophagectomy, evaluating the technical safety and feasibility through the analysis of shortterm outcomes as perioperative mortality, complications, oncological radicality, postoperative pain and cosmetic results.

\section{Methods}

The prospectively collected clinical data of 12 patients who underwent Uniportal VATS esophagectomy and reconstruction with a stomach conduit, from December 2016 to November 2017, at the Department of Thoracic Surgery of Fondazione Policlinico "A. Gemelli" (Rome, Italy), were retrospectively reviewed.

All patients had a diagnosis of upper, middle or lower thoracic esophageal carcinoma.

The preoperative diagnostic — stadiative pathway included: esophagogastroduodenoscopy (EGD) for performing biopsies and endoscopic ultrasound (EUS), Total-body computed tomography (CT) and PET-CT for stadiation of disease, cardiac tests and blood chemistry analyses.

Therapeutic strategies for esophageal cancer can vary according to localization, size and depth of invasion of the tumor, and often indications for neo-adjuvant/adjuvant treatment and type of surgery change among institutions. In our experience and in agreement with recent guide-lines (3), patients with a IIB-IIIB stage [according to the $7^{\text {th }}$ Edition of American Joint Committee on Cancer (AJCC) TNM staging system] underwent preoperative inductive radiochemotherapy (16).

Preoperative re-evaluation and stadiation was carried out by a new PET-CT and EUS when necessary.

All patients signed an informed consent before the operation for the treatment of their clinical data. 


\section{Surgical technique}

Radical surgery for thoracic esophageal carcinoma is accomplished ordinarily as a combination of three approaches: cervical, thoracic and abdominal approach, according to McKeown Technique (17). For reconstruction, the posterior mediastinal route is the most employed and the stomach is the most frequently used organ compared to colon or jejunum.

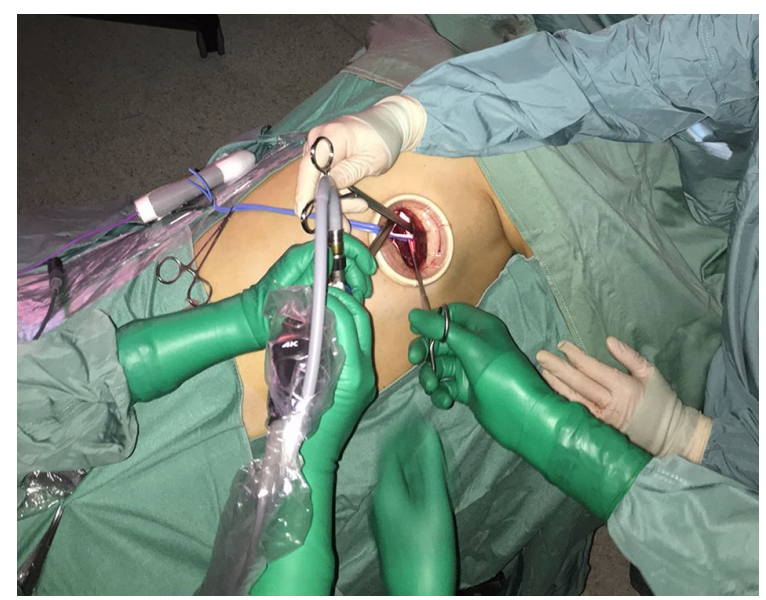

Figure 1 Incision in uniportal VATS esophagectomy. VATS, video-assisted thoracic surgery.
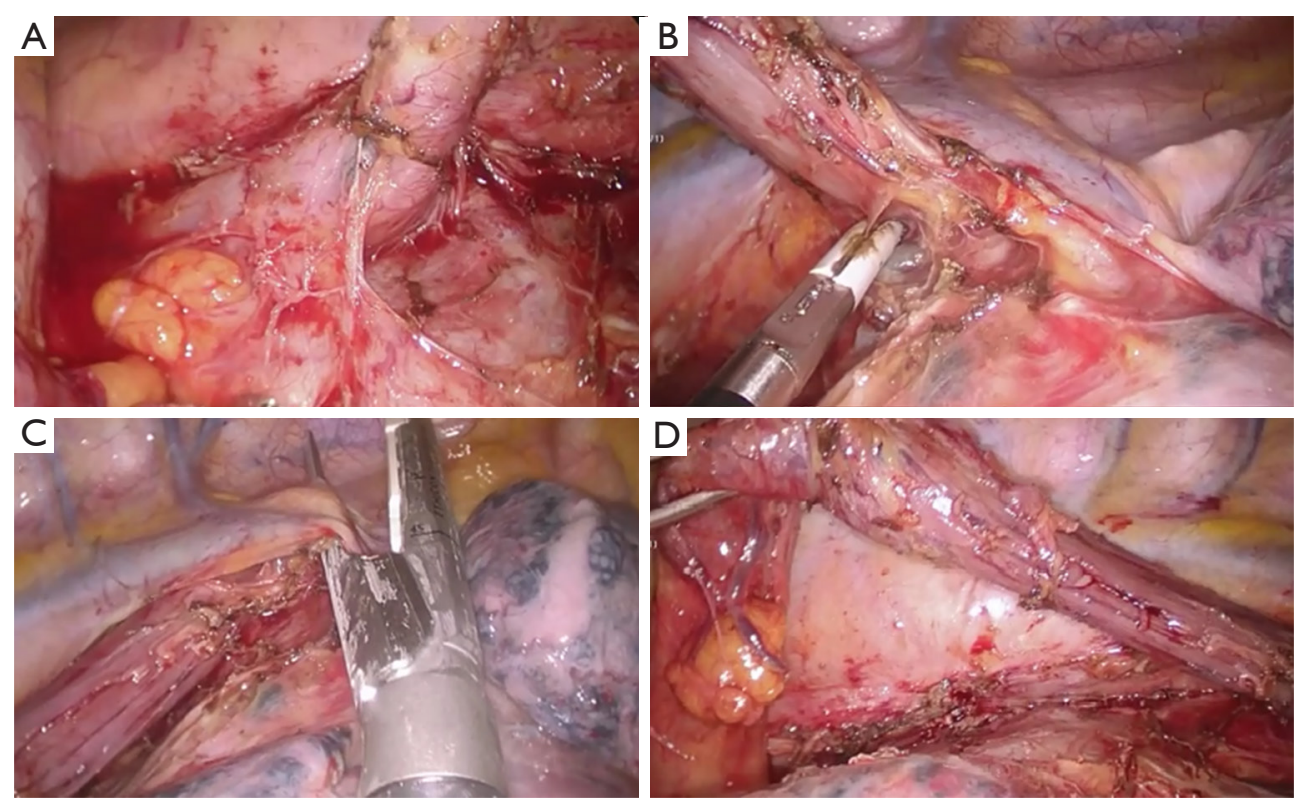

Figure 2 Consecutive steps in uniportal VATS esophagectomy. Dissection and mobilization of the esophagus from the hiatus (A) to the upper part (B). Dissection of azygos vein by stapler (C). Uniportal view of whole esophagus mobilized (D). VATS, video-assisted thoracic surgery. 


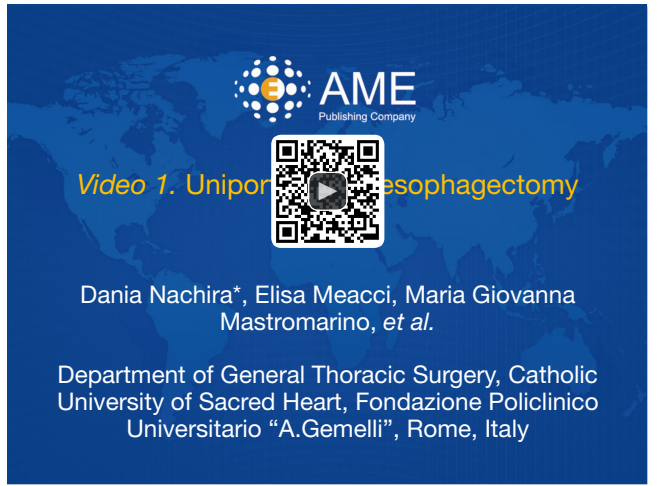

Figure 3 Uniportal VATS esophagectomy (18). VATS, videoassisted thoracic surgery.

Available online: http://www.asvide.com/article/view/28185

was made through an upper median laparotomy.

The right gastroepiploic vessels are preserved and the stomach conduit is made along the greater curvature, by cutting away the cardias and the lesser curvature using endostapler. A pyloroplasty was usually conducted, and a complete abdominal lymphadenectomy (including the celiac axis, common hepatic, left gastric and distal esophagus stations) was performed.

Then the stomach conduit was pulled-up through the esophageal hiatus, along the posterior mediastinum, after taking the native esophagus out (Figure 4), and it was anastomosed in the neck to the cervical stump of native esophagus.

A jejunostomy tube was placed to administer an early enteral nutrition.

\section{Cervical approach}

A small incision was performed on the left side of the neck, along the anterior margin of sternocleidomastoideus muscle. The side-to-side esophagogastric anastomosis, according to the method introduced by Orringer et al. (20), was performed.

\section{Perioperative management}

In the immediate postoperative period, an early mobilization of the patient was incentivized in order to achieve a faster general recovery. An early implementation of enteral nutrition delivered by jejunostomy tube was also administrated and it was managed by a nutritional support team. Indeed, it was reported that enteral nutrition in the perioperative

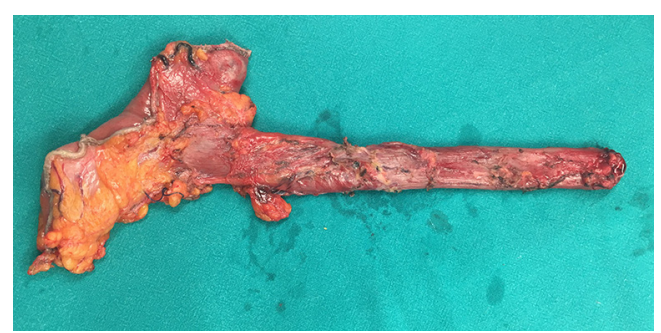

Figure 4 Surgical specimen of esophagus en-bloc with cardias and upper gastric pole.

period decreased the incidence of complications, due to reduced production of endotoxins and inflammatory cytokines, as compared to parenteral nutrition (3). In $\mathrm{V}$ postoperative day an $\mathrm{X}$-ray esophagogram was carried out for evaluating the transit of swallow and excluding anastomotic leak, before restarting oral intake. In our practice, we used to remove the chest drain after the execution of this exam.

\section{Statistical analysis}

Continuous variables were expressed as mean and standard deviation.

Pearson $\chi^{2}$ test and Fischer's exact test were used to compare discrete variables and Student's $t$-test to compare means between two continuous variables.

Univariate analysis was carried out for evaluating potential risk factors in the development of postoperative complications.

A P value less than 0.05 was considered statistically significant.

Statistical analysis was performed using PASW Statistics for Windows, Version 18.0 (SPSS Inc., Chicago, IL, USA).

\section{Results}

The mean age of the population was $60.67 \pm 8.61$ years.

The $83.3 \%$ of the patients were male, with a previous clinical story positive for hiatal hernia in 3 (25\%) cases and a Barrett's esophagus in 1 (8.3\%). The $100 \%$ of population complained dysphagia as main symptom, associated to weight loss in $91.7 \%$ of patients (11 cases).

The main clinic-pathological characteristics of the population are summarized in Table 1.

Seven $(58.3 \%)$ patients underwent preoperative radiochemotherapy because of a locally advanced tumor (cT3N0 or clinical stage III and IVa for only nodal involvement). 
Table 1 Demographical and clinical characteristics of the patients

\begin{tabular}{lc}
\hline Characteristics & $\mathrm{N}=12$ \\
\hline Age (years) & $60.67 \pm 8.61$ \\
Gender (male) & $10(83.3 \%)$ \\
Smoking & $3(25.0 \%)$ \\
COPD & $8(66.7 \%)$ \\
Heart disease & $2(16.7 \%)$ \\
Arterial hypertension & $7(58.3 \%)$ \\
Diabetes & $2(16.7 \%)$ \\
ASA score & $2.08 \pm 0.29$ \\
Other diseases & $4(33.3 \%)$ \\
Hiatal hernia & $3(25.0 \%)$ \\
Barrett's esophagus & $1(8.3 \%)$ \\
Preoperative dysphagia & $12(100.0 \%)$ \\
Weight loss & $11(91.7 \%)$ \\
Neoadjuvant RT/CT & $7(58.3 \%)$ \\
\hline
\end{tabular}

COPD, chronic obstructive pulmonary disease; ASA score, American Society of Anesthesiologists score.

The main histology was squamous cell carcinoma $(50 \%$ of cases, 6 patients), followed by adenocarcinoma in $41.6 \%$ of cases. The $50 \%$ of patients had a middle esophagus cancer, in the $33.3 \%$ of cases the tumor was localized in the upper esophagus and in the $16.7 \%$ in the lower one (Table 2).

The mean operative time of Uniportal VATS esophagectomy was $104.67 \pm 20.66 \mathrm{~min}$ and the mean number of thoracic nodes removed was $10.44 \pm 3.94$. There was no conversion. Chest drain was removed $11.36 \pm 8.75$ days after the operation, after an esophagogram negative for leakage and possible resolution of any other intrathoracic complication, like chylothorax.

The postoperative stay was $15.73 \pm 14.29$ days, with a median value of 9 days. Indeed, the postoperative onset of some complications (Table 3) caused a lengthening of the hospital stay, in some cases: $2(16.7 \%)$ patients developed an anastomotic leak (treated in a conservative way), with a consequent pneumonia, and $1(8.3 \%)$ patient a chylothorax (which required a surgical treatment).

The incidence of postoperative complications was not significantly correlated to any potential risk factor analyzed (like age, gender, comorbidities, weight loss, localization of the tumor, stage, neoadjuvant treatment...).

The main pathological features of the tumor are reported in Table 2. All esophagectomies performed were radical, achieving a R0 status. In the postoperative period, 8 (66.7\%) patients were referred to adjuvant treatment.

No patient had a local recurrence, and after $4.33 \pm 3.31$ (range, $1-11)$ months 10 patients $(83.3 \%)$ had no evidence of disease and $10(83.3 \%)$ patients were alive; 2 (16.7\%) patients died of other causes not related to cancer (Table 4).

Outcomes in terms of postoperative pain and cosmetic results were satisfactory (Table 4). The mean level of pain measured on VAS scale in first postoperative day was $1.92 \pm 0.90$, with a mean duration of $2.25 \pm 1.54$ days. All patients reported a resolution of pain after chest drain removal and no one developed postoperative paresthesia and neuralgia. Cosmetic result was valued on a 3-point scale and the mean value was $2.42 \pm 0.79$. Furthermore, no wound infection was recorded.

\section{Discussion}

Surgery remains the cornerstone in esophageal cancer treatment, although esophagectomy is burdened by a relatively high morbidity rate, with mortality ranging from $1.4 \%$ in high-volume centers (6) to $23 \%$ in low-volume centres (21). To reduce the incidence of complications, since 1990s MIE has been introduced, and the study of Cuschieri and colleagues (5) revealed that MIE was as safe and effective as $\mathrm{OE}$. The first minimally invasive technique was performed by Depaula et al. (22) and it consisted of a totally laparoscopic approach with cervical anastomosis. However, the first thoracoscopic esophageal mobilization was credited to Cuschieri (23) in 1994. Since then, many technical improvements and modifications have occurred. Nowadays, the most widely adopted technique is the combination between the thoracoscopic approach, in the left lateral decubitus position, followed by laparoscopic or upper median laparotomic creation of a gastric conduit with a cervical anastomosis (6). In the last two decades MIE has shown an improved short-term outcome characterized by a lower incidence of respiratory complications, a shorter hospital stay, a lower blood loss and better short-term quality of life compared with $\mathrm{OE}$, with no compromise in the quality of surgical resection $(7,8,24-26)$. Indeed, MIE should reproduce the same technical procedure performed in the standard $\mathrm{OE}$ and it should follow the same oncologic principles consisting of a complete, radical R0, en-bloc esophagectomy associated with an extended 2-field lymphadenectomy.

Biere and colleagues (8) published the first randomized, 
Table 2 Intra-, post-operative and pathological results

\begin{tabular}{|c|c|}
\hline Variables & $\mathrm{N}=12$ \\
\hline Operative time (min) & $104.67 \pm 20.66$ \\
\hline Conversion & 0 \\
\hline Chest drain removal (days) & $11.36 \pm 8.75$ \\
\hline Post-operative stay (days) & $15.73 \pm 14.29$ \\
\hline \multicolumn{2}{|l|}{ Localization of the tumor } \\
\hline Upper thoracic esophagus & $4(33.3 \%)$ \\
\hline Middle thoracic esophagus & $6(50.0 \%)$ \\
\hline Lower thoracic esophagus & $2(16.7 \%)$ \\
\hline \multicolumn{2}{|l|}{ Histology } \\
\hline Adenocarcinoma & $5(41.6 \%)$ \\
\hline Squamous cell carcinoma & $6(50.0 \%)$ \\
\hline Adenoid cystic carcinoma & $1(8.4 \%)$ \\
\hline Tumor extension (cm) & $9.52 \pm 12.36$ \\
\hline \multicolumn{2}{|l|}{ Stage } \\
\hline Complete response & $2(16.7 \%)$ \\
\hline $1 \mathrm{~A}$ & $1(8.3 \%)$ \\
\hline $2 \mathrm{~A}$ & $3(25.0 \%)$ \\
\hline 2B & $3(25.0 \%)$ \\
\hline $3 A$ & $1(8.3 \%)$ \\
\hline 3B & $1(8.3 \%)$ \\
\hline $3 C$ & $1(8.3 \%)$ \\
\hline \multicolumn{2}{|l|}{ Grading } \\
\hline G2 & $6(50.0 \%)$ \\
\hline G3 & $4(33.3 \%)$ \\
\hline \multicolumn{2}{|l|}{$\begin{array}{l}\text { Mandard regression grade (for } 7 \text { patients } \\
\text { underwent Neoadjuvant therapy) }\end{array}$} \\
\hline TRG1 & $2(16.7 \%)$ \\
\hline TRG2 & $1(8.3 \%)$ \\
\hline TRG3 & $3(25 \%)$ \\
\hline TRG4 & $1(8.3 \%)$ \\
\hline Number of thoracic lymph nodes removed & $10.44 \pm 3.94$ \\
\hline Positive thoracic lymph nodes & $0.66 \pm 1.66$ \\
\hline R-status + & 0 \\
\hline Adjuvant treatment & $8(66.7 \%)$ \\
\hline
\end{tabular}

Table 3 Post-operative complications

\begin{tabular}{lc}
\hline Complications & $\mathrm{N}=12$ \\
\hline Anastomotic leak & $2(16.7 \%)$ \\
Anemia & $1(8.3 \%)$ \\
Chylothorax & $1(8.3 \%)$ \\
Atrial fibrillation & $4(33.3 \%)$ \\
Pneumonia & $2(16.7 \%)$ \\
\hline
\end{tabular}

Table 4 Postoperative outcomes

\begin{tabular}{lc}
\hline Variables & $\mathrm{N}=12$ \\
\hline Local recurrence & 0 \\
Overall survival (months) & $4.33 \pm 3.31[1-11]$ \\
Dead of disease & 0 \\
Dead of other causes & $2(16.7 \%)$ \\
Alive with disease & 0 \\
Non evidence of disease & $10(83.3 \%)$ \\
Pain in first postoperative day (VAS scale) & $1.92 \pm 0.90$ \\
Mean duration of pain (days) & $2.25 \pm 1.54$ \\
Pain after chest tube removal (YES) & 0 \\
Pain after chest tube removal (VAS scale) & 0 \\
Cosmetic result (1 to 3 points) & $2.42 \pm 0.79$ \\
Postoperative paresthesia and neuralgia & 0 \\
Wound infections & 0 \\
\hline
\end{tabular}

multicentre trial showing short-term benefits of MIE for patients with resectable esophageal cancer. In particular, in TIME trial MIE resulted in a decrease of blood loss and postoperative pulmonary infections, a faster postoperative recovery with an improvement of health-related quality of life. These results were associated with no difference in 30-day mortality and specially in oncological outcome, as assessed by no difference in the number of retrieved lymph nodes and the completeness of resection. Similar results were achieved in a prospective randomized study conducted by Guo et al. (24) and they were confirmed in two recent meta-analyses $(7,26)$. Nagpal and colleagues (26) analysed postoperative outcomes, including anastomotic leak, anastomotic stricture, gastric conduit ischemia, chylothorax, and recurrent laryngeal paralysis and they concluded that there was no significant difference between MIE compared 
with OE. Furthermore, although there was no change in 30-day mortality, patients who underwent MIE may benefit from lower blood loss, shorter hospital stay, and reduced total morbidity and respiratory complications without compromising lymph node clearance and margin of resection. Lv et al. (7) reached the same conclusions in their study.

While the advantages of MIE in comparison with open thoracotomy were already proven, controversy has been still existing about the best patient's surgical position, if prone or left lateral decubitus. The first thoracoscopic esophageal mobilization was described in 1994 by Cuschieri (23) in prone position but, after this experience, lateral decubitus technique grew to the point to be the main method adopted worldwide currently (6). However, more recently, there has been a shift in some Western surgical groups $(11,13,27)$ in favour of the prone technique. These authors affirmed an advantage in thoracoscopic operative time, which is faster compared to that one of left lateral decubitus, but the overall operative time is generally not shorter in the prone procedure, because the patient's repositioning is more difficult and time consuming. Moreover this technique has shown comparable outcomes to the lateral position, in terms of intraoperative blood loss, number of lymph nodes dissected, length of stay and complications (11). Thus, no clear benefits have been proven in prone position, while, according to experience and literature reports (13) disadvantages are represented by difficulties in airway control for anesthesiological team and in conversion to open, especially in emergency. Indeed, although a posterior thoracotomy can be performed in this position, it is a less familiar surgical approach and can be difficult. An intermediate technique to overcome these disadvantages is the semi-prone position performed by Bonavina and colleagues (12).

Surgical port-site creation represents another matter of debate; the multiportal approach is the standardized procedure largely performed worldwide $(8,12,13)$; conversely no certain evidence has been in literature about the feasibility of thoracoscopic esophageal mobilization in uniportal VATS till now. Although few technical papers (15) and a study about preliminary results of single-incision MIE have been published previously (14), the safety and the effectiveness of this new approach remain to be demonstrated. Lee et al. (14), in their propensity-match study comparing perioperative outcome in MIE performed with single or multi-incision approach, reported similar perioperative results, in terms of postoperative ICU and length of hospital stay, number of lymph nodes retrieved and incidence of major surgical complications between the two groups of patients. Moreover, single-incision MIE provided a significantly lower pain one week after surgery.

The uniportal VATS approach has gradually been adopted and nowadays it is a well-established technique in pulmonary surgery (28). With the experience accumulated in this field, we started Uniportal VATS esophagectomy since December 2016 in our institution. The single $4-5 \mathrm{~cm}$ incision in left lateral decubitus position allows surgeon to apply the same principle as when performing an anterolateral thoracotomy in open surgery, that means having a direct view of the operating field. Also the surgeon's wrists are in a neutral position in relation to the forearms, minimizing fatigue and maximizing ergonomic function. Furthermore, the reverse-Trendelenburg position and the anterior rotation of the table $30-45^{\circ}$ toward the surgeon facilitate lung displacement necessary to perform the mobilization of the esophagus. The Uniportal technique may also avoid the risk of injury of the intercostal neurovascular bundle. Another incontestable advantage that must be considered it is the ease of conversion to open if required, either electively or emergently, due to the patient's position that is already in lateral decubitus and the fact that it is sufficient prolonging the incision on $\mathrm{V}$ intercostal space to perform a lateral thoracotomy. In our experience there was no conversion.

The training for esophagectomy in uniportal VATS can be long but, thanks to the acquired practice in uniportal VATS lung surgery, the mean thoracoscopic operative time $(104.67 \pm 20.66 \mathrm{~min})$ was comparable to that reported for multiportal approach $(8,11)$, with a postoperative complication rate similar to data presented in literature $(7,26)$. In particular, in our series, the only two cases of pneumonia were observed subsequently to anastomotic leakage in the neck, therefore not related to ischemiareperfusion injury and oxidative stress (29) due to a prolonged one-lung ventilation needed in longer operative time.

From the oncological standpoint, concerns have been still existing regarding minimally invasive surgery in the setting of cancer. In our series we noted no compromise in the quality of the resected specimen, in fact all patients underwent $\mathrm{R} 0$ resections, without evidence of local recurrence. The number of lymph nodes retrieved is important for an accurate nodal staging and related prognosis of esophageal cancer (30); we observed a mean number of thoracic lymph nodes removed during 
esophagectomy of $10.44 \pm 3.94$, in line with other published reports $(7,8,11)$. This suggests that uniportal VATS approach allows comparable dissection to multiportal MIE and $\mathrm{OE}$ without compromising oncological outcome.

In according to recent guide-lines (3), neoadjuvant chemoradiation represents the standard care before surgery for locally advanced esophageal cancer and the efficacy of MIE has been demonstrated even in patients who have received this treatment $(8,31)$. About $58 \%$ of our patients underwent preoperative radio-chemotherapy because of a locally advanced tumor; in this group the operative time and the number of dissected lymph nodes were similar to the group of patients without previous inductive treatment. Therefore, Uniportal VATS esophagectomy can be an effective approach to perform esophageal mobilization and lymphadenectomy after neoadjuvant chemoradiation.

Even if we observed no significant correlation between the incidence of postoperative complications and any potential risk factor analyzed (like age, gender, comorbidities, weight loss, stage...) we found a trend of major complications in patients who underwent neoadjuvant treatment. Indeed, both the two anastomotic leaks and the one chylothorax occurred in patients who have received previous induction treatment. These complications seemed not related to single-incision procedure but perhaps they could be related the effects of radio-chemotherapy on tissues and general comorbidities of the patients. The onset of chylothorax could be explained by the remodeling of tissues caused by radiationinduced fibrosis which makes thoracic duct identification more difficult. Radiation damage to gastric fundus vascularization could also increase the risk of anastomotic leakage in these patients. However, a no negligible bias was represented by the very small sample size.

Referring to the only report (14) which compared singleand multi-incision MIE, we noted a lower postoperative hospital stay with a median value of 9 days $(15.73 \pm 14.29$ days in our group versus $20.90 \pm 8.9$ days in single-incision Lee's group). A lengthening of hospitalization was caused by the onset of major complications which required prolonged postoperative treatments.

MIE is also associated with a better quality of life compared with OE (25); in particularly the lower postoperative wound pain, related to the less invasive nature of the operation, plays a key role in decreasing respiratory complications. This advantage is more evident in Uniportal approach, due to reduced number of port sites minimizing the risk of intercostal nerves injury, as reported in Taiwan group experience (14). In our series a great benefit has been made by the intercostal nerve blockade by ropivacaine infiltration under endoscopic view performed during thoracoscopic approach (19). We observed a positive impact on postoperative pain, with a mean of $2.25 \pm 1.54$ days, which resulted in a less need for postoperative analgesia and an early patient mobilization, thereby facilitating a faster general recovery. Additionally, a multimodality treatment plan including early postoperative physiotherapy and enteral nutrition increased the possibility of a quick recovery. Indeed, it is reported that enteral support in the perioperative period decreases the incidence of complications, due to reduced production of endotoxins and inflammatory cytokines, as compared to parenteral nutrition (3).

Nevertheless, cosmetic results should be considered in oncologic patients. An optimal cosmetic outcome can be achieved with the uniportal approach; on a 3-point subjective scale, the mean value of satisfaction observed was $2.42 \pm 0.79$.

This study presents likewise some limitations. In the first place, it is a single centre, retrospective, non randomised series affected by selection biases. In the second place, the very small sample size, without a control group, may reduce the effectiveness of our results. Finally, a longer oncological follow-up would be needed to demonstrate the safety of this technique in terms of long-term outcome. However, to the best of our knowledge, this is the first published paper about uniportal VATS esophagectomy which reports preliminary results concerning postoperative and oncological outcomes.

Uniportal VATS esophagectomy seems to be a safe, feasible and effective technique which appears to be equivalent to multiportal approach in terms of operative time, postoperative morbidity and mortality, short length of hospital stay and oncological outcome. Early relief from postoperative pain, fast recovery and cosmetic results are also important advantages observed in this technique. Nevertheless future prospective randomized controlled trials with a satisfactory oncological followup should be performed to confirm these preliminary results and to demonstrate the effectiveness of Uniportal VATS esophagectomy in improving short- and long-term outcomes in patients with resectable esophageal cancer.

\section{Acknowledgements}

None. 


\section{Footnote}

Conflicts of Interest: The authors have no conflicts of interest to declare.

Ethical Statement: This study was evaluated by the Institutional Review Board (IRB) of Catholic University of Sacred Hearth and, as this was a retrospective review for service evaluation (within an audit approved by our Surgical Department) and there was no modification in patients' care (no prospective randomized study), we did not need the final ethical approval of our IRB. All patients signed an informed consent before the operation for the treatment of their clinical data.

\section{References}

1. Pennathur A, Gibson MK, Jobe BA, et al. Oesophageal carcinoma. Lancet 2013;381:400-12.

2. Zhang Y. Epidemiology of esophageal cancer. World J Gastroenterol 2013;19:5598-606.

3. Kuwano H, Nishimura Y, Oyama T, et al. Guidelines for Diagnosis and Treatment of Carcinoma of the Esophagus April 2012 edited by the Japan Esophageal Society. Esophagus 2015;12:1-30.

4. Hulscher JB, van Sandick JW, de Boer AG, et al. Extended transthoracic resection compared with limited transhiatal resection for adenocarcinoma of the esophagus. $\mathrm{N} \mathrm{Engl} \mathrm{J}$ Med 2002;347:1662-9.

5. Cuschieri A, Shimi S, Banting S. Endoscopic oesophagectomy through a right thoracoscopic approach. J R Coll Surg Edinb 1992;37:7-11.

6. Luketich JD, Alvelo-Rivera M, Buenaventura PO, et al. Minimally invasive esophagectomy: outcomes in 222 patients. Ann Surg 2003;238:486-94; discussion 494-5.

7. Lv L, Hu W, Ren Y, et al. Minimally invasive esophagectomy versus open esophagectomy for esophageal cancer: a meta-analysis. Onco Targets Ther 2016;9:6751-62.

8. Biere SS, van Berge Henegouwen MI, Maas KW, et al. Minimally invasive versus open oesophagectomy for patients with oesophageal cancer: a multicentre, open-label, randomised controlled trial. Lancet 2012;379:1887-92.

9. Smithers BM, Gotley DC, Martin I, et al. Comparison of the outcomes between open and minimally invasive esophagectomy. Ann Surg 2007;245:232-40.

10. Park S, Hwang Y, Lee HJ, et al. Comparison of robotassisted esophagectomy and thoracoscopic esophagectomy in esophageal squamous cell carcinoma. J Thorac Dis 2016;8:2853-61.

11. Fabian T, Martin J, Katigbak M, et al. Thoracoscopic esophageal mobilization during minimally invasive esophagectomy: a head-to-head comparison of prone versus decubitus positions. Surg Endosc 2008;22:2485-91.

12. Bonavina L, Asti E, Sironi A, et al. Hybrid and total minimally invasive esophagectomy: how I do it. J Thorac Dis 2017;9:S761-72.

13. Depypere L, Coosemans W, Nafteux P, et al. Videoassisted thoracoscopic surgery and open chest surgery in esophageal cancer treatment: present and future. J Vis Surg 2017;3:30.

14. Lee JM, Chen SC, Yang SM, et al. Comparison of singleand multi-incision minimally invasive esophagectomy (MIE) for treating esophageal cancer: a propensitymatched study. Surg Endosc 2017;31:2925-31.

15. Dmitrii S, Pavel K. Uniportal Video-Assisted Thoracic Surgery Esophagectomy. Thorac Surg Clin 2017;27:407-15

16. Edge SB, Compton CC. The American Joint Committee on Cancer: the 7th edition of the AJCC cancer staging manual and the future of TNM. Ann Surg Oncol 2010;17:1471-4.

17. McKeown KC. Total three-stage oesophagectomy for cancer of the oesophagus. Br J Surg 1976;63:259-62.

18. Nachira D, Meacci E, Mastromarino MG, et al. Uniportal VATS esophagectomy. Asvide 2018;5:844. Available online: http://www.asvide.com/article/view/28185

19. Ismail M, Swierzy M, Nachira D, et al. Uniportal videoassisted thoracic surgery for major lung resections: pitfalls, tips and tricks. J Thorac Dis 2017;9:885-97.

20. Orringer MB, Marshall B, Iannettoni MD. Eliminating the cervical esophagogastric anastomotic leak with a sideto-side stapled anastomosis. J Thorac Cardiovasc Surg 2000;119:277-88.

21. Birkmeyer JD, Siewers AE, Finlayson EV, et al. Hospital volume and surgical mortality in the United States. N Engl J Med 2002;346:1128-37.

22. DePaula AL, Hashiba K, Ferreira EA, et al. Laparoscopic transhiatal esophagectomy with esophagogastroplasty. Surg Laparosc Endosc 1995;5:1-5.

23. Cuschieri A. Thoracoscopic subtotal oesophagectomy. Endosc Surg Allied Technol 1994;2:21-5.

24. Guo M, Xie B, Sun X, et al. A comparative study of the therapeutic effect in two protocols: video-assisted thoracic surgery combined with laparoscopy versus right open transthoracic esophagectomy for esophageal cancer management. Chin German J Clin Oncol 2013;12:68-71. 
25. Maas KW, Cuesta MA, van Berge Henegouwen MI, et al. Quality of Life and Late Complications After Minimally Invasive Compared to Open Esophagectomy: Results of a Randomized Trial. World J Surg 2015;39:1986-93.

26. Nagpal K, Ahmed K, Vats A, et al. Is minimally invasive surgery beneficial in the management of esophageal cancer? A meta-analysis. Surg Endosc 2010;24:1621-9.

27. Palanivelu C, Prakash A, Senthilkumar R, et al. Minimally invasive esophagectomy: thoracoscopic mobilization of the esophagus and mediastinal lymphadenectomy in prone position--experience of 130 patients. J Am Coll Surg 2006;203:7-16.

28. Gonzalez-Rivas D, Fieira E, Mendez L, et al. Single-port video-assisted thoracoscopic anatomic segmentectomy

Cite this article as: Nachira D, Meacci E, Mastromarino MG, Pogliani L, Zanfrini E, Iaffaldano A, Petracca-Ciavarella L, Chiappetta M, Congedo MT, Vita ML, Porziella V, Margaritora S. Initial experience with uniportal video-assisted thoracic surgery esophagectomy. J Thorac Dis 2018;10(Suppl 31):S3686-S3695. doi: 10.21037/jtd.2018.04.17 and right upper lobectomy. Eur J Cardiothorac Surg 2012;42:e169-71.

29. Cheng YJ, Chan KC, Chien CT, et al. Oxidative stress during 1-lung ventilation. J Thorac Cardiovasc Surg 2006;132:513-8.

30. Gu Y, Swisher SG, Ajani JA, et al. The number of lymph nodes with metastasis predicts survival in patients with esophageal or esophagogastric junction adenocarcinoma who receive preoperative chemoradiation. Cancer 2006;106:1017-25.

31. Warner S, Chang YH, Paripati H, et al. Outcomes of minimally invasive esophagectomy in esophageal cancer after neoadjuvant chemoradiotherapy. Ann Thorac Surg 2014;97:439-45. 